\title{
Masculinité, mensonge, Jeckischkeit dans le roman de Yoram Kaniuk, 1948
}

Männlichkeit, Lüge, "Jeckischkeit" in Yoram Kaniuks Roman 1948

Masculinity, lie, 'Yekkishness' in Yoram Kaniuk's novel 1948

\section{Patrick Farges}

\section{(2) OpenEdition}

\section{Journals}

Édition électronique

URL : http://journals.openedition.org/ceg/1585

DOI : $10.4000 /$ ceg. 1585

ISSN : 2605-8359

\section{Éditeur}

Presses Universitaires de Provence

Édition imprimée

Date de publication : 15 juin 2015

Pagination : 219-228

ISBN : 978-2-85399-993-9

ISSN : 0751-4239

\section{Référence électronique}

Patrick Farges, « Masculinité, mensonge, Jeckischkeit dans le roman de Yoram Kaniuk, 1948 », Cahiers d'Études Germaniques [En ligne], 68 | 2015, mis en ligne le 17 décembre 2017, consulté le 01 décembre 2020. URL : http://journals.openedition.org/ceg/1585; DOI : https://doi.org/10.4000/ceg.1585 


\section{Masculinité, mensonge, Jeckischkeit dans le roman de Yoram Kaniuk, 1948}

Patrick FARGES

Université Sorbonne Nouvelle - Paris 3

\section{Introduction : masculinité, mensonge, narration}

Le présent article se place dans la continuité des travaux ayant démontré un intérêt renouvelé pour les liens entre mensonge et narration. Le colloque d'AixMarseille en octobre 2013 a ainsi permis d'interroger la sémantique du mensonge. Par ailleurs, les approches narratologiques récentes faisant une place aux questions de genre ${ }^{1}$ et d'intersectionnalité ont également ouvert la voie à une interrogation sur le mensonge: c'est le cas, par exemple, de la revue des narratologues de l'université de Wuppertal, Diegesis. Interdisziplinäres E-Journal für Erzählforschung, qui consacre un numéro spécial (le n ${ }^{\circ} 4.1$, à paraître en 2015) au thème «Erzählen und Lüge ${ }^{2} »$. La sémantique du mensonge affecte la construction du masculin et du féminin de manière différentielle, et reflète ainsi la «valence différentielle des sexes » mise en avant par l'anthropologue Françoise Héritier ${ }^{3}$. Avec Judith Butler, on peut par ailleurs affirmer que la performance de quelque norme de genre que ce soit, tant féminine que masculine, passe par l'imitation d'une imitation, et donc par la performance récurrente d'une forme de simulation ${ }^{4}$. Il s'agit donc bien d'une « comédie » de genre, notamment pour la masculinité. « On ne naît donc pas homme, on le devient », affirme ainsi l'historienne Anne-Marie Sohn paraphrasant la célèbre formule de Beauvoir ${ }^{5}$.

Dans le cadre d'une interrogation genrée sur le masculin et la masculinité,

Sur les liens entre narration et genre, cf. Patrick Farges, Cécile Chamayou-Kunn et Perin Emel Yavuz, Le Lieu du genre. La narration comme espace performatif du genre, Paris, Presses Sorbonne Nouvelle, 2011.

2 https://www.diegesis.uni-wuppertal.de/index.php/diegesis (dernière consultation: 2 septembre 2014).

3 Cf. Françoise Héritier, Masculin-Féminin I. La Pensée de la différence, Paris, Odile Jacob, 1996; Françoise HérITIER, Masculin-Féminin II. Dissoudre la hiérarchie, Paris, Odile Jacob, 2002.

${ }_{4}^{4}$ Judith ButLer, Trouble dans le genre. Le féminisme et la subversion de l'identité [1990], trad. Cynthia Kraus, Paris, La Découverte, 2006.

5 Anne-Marie SoHN, «Sois un homme! ». La construction de la masculinité au XIXe siècle, Paris, Seuil, 2009, p. 8. 
il semble pertinent de s'interroger sur le rapport des hommes et du masculin au mensonge et à la dis/simulation. En effet, il faut pouvoir interroger l'un des stéréotypes qui s'est installé progressivement à partir du XVIII ${ }^{\mathrm{e}}$ siècle par l'apparition de discours normatifs sur le sexe et le genre, stéréotype selon lequel les hommes maîtriseraient le mensonge alors que les femmes seraient le mensonge ${ }^{6}$. Par ailleurs, parmi les mensonges récalcitrants, pérennes, il y a celui qui veut que la masculinité soit invisible parce qu'allant de soi, celui qui veut que la masculinité soit l'apanage des hommes uniquement ${ }^{7}$ ou encore celui selon lequel la masculinité serait «en crise », alors même que le motif de la crise sert bien souvent à réaffirmer l'hégémonie en proposant un nouvel arrangement de domination ${ }^{8}$.

Dans le présent article, je me propose de réinterroger les masculinités juives-allemandes à l'aune du mensonge, et en lien avec le mythe national sioniste, lui-même fortement masculinisé. Cette interrogation, qui s'intègre dans un projet plus large concernant les masculinités juives-allemandes en Palestine sous mandat britannique puis en Israël dans la post-migration ${ }^{9}$, sera ici appliquée au dernier roman, intitulé 1948, de l'auteur israélien Yoram Kaniuk, né en 1930 et décédé en juin $2013{ }^{10}$. Il s'agira dans un premier temps d'interroger le statut de ce texte, qui hésite entre fiction et littérature testimoniale. Dans un second temps, il s'agira d'interroger la manière dont s'est cristallisé un mensonge de genre qui a conduit - notamment à l'occasion du moment-charnière qu'est la guerre de 1948 -à magnifier une vision héroïque et virile - préexistante - du « Juif nouveau ». Enfin, j’étudierai la dimension de « roman familial » (et notamment la relation père-fils), qui conduit à dénoncer une forme de masculinité qui peut paraître alternative: la masculinité juive-allemande (yekke dans l'usage israélien) cultivée, contenue et bourgeoise.

\section{Un roman-témoignage? Le statut du texte}

Il convient d'abord de s'interroger sur le statut de ce texte, à mi-chemin entre œuvre de fiction et témoignage. C'est sans doute la nature de l'événement même, « 1948 »

6 Sur la permanence de stéréotypes de genre, voir le lexique d'Ilana Löwy et Catherine MARry, Pour en finir avec la domination masculine. De A à Z, Paris, Seuil (Les Empêcheurs de penser en rond), 2007.

7 Pour une déconstruction de cela, on se reportera à Judith Halberstam, Female Masculinity, Durham/ Londres, Duke University Press, 1998; Marie-Hélène Bourcier et Pascale Molinier (dir.), Les fleurs du mâle: masculinités sans hommes? (Cahiers du genre, $\mathrm{n}^{\circ}$ 45), Paris, L'Harmattan, 2008.

8 Voir à ce sujet Pascale Molinier, « Déconstruire la crise de la masculinité », Mouvements, $\mathrm{n}^{\circ} 31.1$, 2004, p. 24-29.

9 Sur le projet, voir Patrick Farges, « Exilerfahrung und Refiguration von Männlichkeitskonzepten. Eine neue Perspektive auf das Israel-Korpus », in Doerte Bischoff, Susanne Komfort-HeIn (dir.), Literatur und Exil. Neue Perspektiven, Berlin/ Boston, De Gruyter, 2013, p. 257-282; Patrick FARGES, « Männlichkeitskonstruktionen von Jeckes in Israel », Germanistik in der Schweiz, nº 10, 2013, p. 21-30.

${ }^{10}$ Yoram Kaniuk, 1948 [1 ${ }^{\text {re }}$ éd. 2010], trad. Laurence Sendrowicz, Paris, Fayard, 2012. Le roman a reçu une distinction littéraire en Israël, le Prix Sapir. Il été adapté pour la scène du théâtre de Haïfa en 2011. L'auteur israélien n'a pas été beaucoup étudié par les études germaniques françaises, à l'exception notable de la thèse de doctorat de Sophie ZIMmER, « Le renouveau juif à Berlin depuis 1989: aspects culturels et religieux » (université Paris Sorbonne - Paris IV, thèse soutenue en 2012), où c'est l'image de Berlin chez Kaniuk qui est principalement interrogée. 
soit la «Guerre d'indépendance » ayant conduit à la création d'Israël comme État juif, qui contribue à brouiller les pistes. En effet, il s'agit là d'un événement fondateur dans l'histoire d'Israël, d'un événement extrêmement controversé également. Comme le rappelle le narrateur: " 1945 a été une année de transition, une année charnière, elle se situe entre l'anéantissement et ce qui nous est apparu comme une avancée décisive dans notre combat contre le destin juif » $\left(52^{11}\right)$. Kaniuk situe son livre au cœur de l'autre événement-charnière de l'histoire juive au XXe siècle: 1948 - qui fournit le titre de son livre (תשח, Tachakh en hébreu), c'est-à-dire le moment où le mythe du nouveau juif pionnier et combattant semble se réaliser pleinement. «Chakh», qui constitue la seconde partie du mot « Tachakh» signifie « [jeu d']échecs », mot avec lequel il partage d'ailleurs l'étymologie: 1948 est donc un moment déterminant de choix, un moment de krisis ${ }^{12}$ où il s'agit de décider vite. C'est aussi le moment du kairos $^{13}$, c'est-à-dire le moment opportun: la proclamation de la naissance d'Israël est d'ailleurs présentée rétrospectivement comme un formidable coup de dés, qui n'est pas perçu comme tel par le narrateur et ses camarades à l'époque. Ils préfèrent dormir, épuisés qu'ils sont par l'âpreté et la précarité de leur situation.

Il y a un « avant » et un « après » 1948: dans le récit historique, ce qui est vérité à un moment devient mensonge l'instant d'après. La nature même de l'événement a donc partie liée avec le mensonge ${ }^{14}$. C'est ce que rappelle le narrateur vers la fin $\mathrm{du}$ roman, à l'évocation de ces « absents-présents", soit les populations arabes ayant fui leur lieu d'habitation durant une période bien précise et qui se sont retrouvées expropriées de fait:

Ce concept a par la suite été pérennisé et inscrit dans les lois israéliennes. [...] Tout Arabe étant sorti d'une ville conquise avant le 14 mai 1948 [...] et qui a voulu revenir après cette date, est considéré comme n'ayant pas été là puisque parti. Il était présent car il avait été là, mais il était absent car il n'était plus là. (229)

Au fil de la narration en focalisation interne, l'expérience virile et héroïque de l'événement « 1948 », qui est fondateur tant d'un point de vue historique (parce qu'il appartient à la « grande » Histoire) que d'un point de vue biographique (parce qu'il correspond aux années formatrices du narrateur, alors âgé de dix-sept ans), est régulièrement dénoncée comme étant un vaste mensonge. Le lecteur se trouve confronté à une forme de paradoxe du menteur, en ce que le narrateur lui-même admet le mensonge. Le narrateur est-il fiable ou non ${ }^{15}$ ? Dès l'incipit, le doute plane:

11 Tous les numéros de page se réfèrent à la traduction française du roman.

${ }^{12}$ Krisis signifie trier, et par extension décider selon des critères (mot qui a la même étymologie) et juger. La crise est donc le moment où l'on distingue afin de séparer; elle s'oppose aux évolutions lentes.

${ }^{13}$ Kairos est le moment furtif qui permet de saisir l'occasion opportune; il s'agit d'un moment propice, qui peut ne pas se représenter. Il est déterminant pour les actions qui doivent être accomplies «à temps », sans atermoiement. Le kairos implique une vision du temps compatible avec une action humaine efficace.

${ }^{14}$ Voir à ce sujet Rochelle Furstenberg, «The Jewish Sabra. An Interview with Novelist Yoram Kaniuk », The Jerusalem Post, 10 juillet 2011, http://www.jpost.com/Jerusalem-Report/Arts-AndCulture/The-Jewish-Sabra (dernière consultation: 4 septembre 2014).

15 Sur la question du narrateur non-fiable, on consultera Gregory CURRIE, « L'interprétation du nonfiable: narrateurs non-fiables et œuvres non-fiables », http://www.vox-poetica.org/t/articles/currie.html (dernière consultation: 3 septembre 2014); Dieter MEINDL, «(Un-)reliable Narration from a Pronominal 
«Que cela ait réellement eu lieu ou non, de cette façon ou d'une autre, aucune mémoire n'a d'État, aucun État n'a de mémoire. Je peux avoir des souvenirs réels ou en inventer [...].» (11) L'imbrication même d'une mémoire personnelle avec une mémoire d'État rend d'emblée la question de la vérité et du mensonge caduque. Cela est d'ailleurs rappelé à plusieurs reprises au fil des pages du roman:

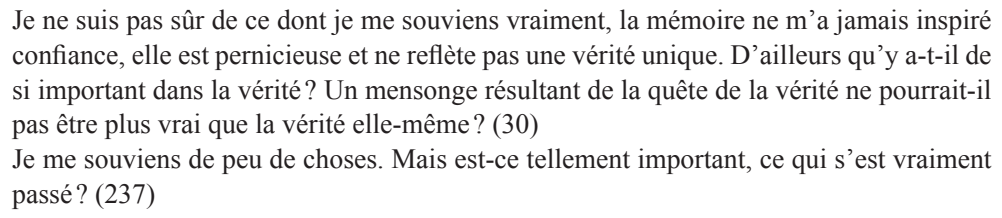

Parfois, le narrateur a même recours à une forme éculée d'humour juif pour réaffirmer qu'il ne peut s'agir de rechercher la vérité: "Aujourd'hui, je suis très vieux, j'écris ces choses mais mon cerveau est vide. Je suis moi-même le trou du bagel.» (161)

Les souvenirs du narrateur sont pris dans des « cadres sociaux » et historiques qui brouillent la question de la vérité. Ces cadres sociaux sont par ailleurs très fortement genrés, ce que la construction nationale (ici: de la nation israélienne) n'a pas démenti, en assignant un rôle de premier plan au « nouvel homme juif ». Ainsi les travaux historiques féministes ont-ils montré à quel point les " pionnières » du projet sioniste avaient été rendues invisibles dans l'historiographie ou bien qu'elles avaient été reléguées, sauf exception, dans des fonctions «féminines » et subalternes ${ }^{16}$. Si l'objectif premier des historiennes féministes depuis les années 1970 a été de faire reconnaître la participation des femmes dans la nation et dans l'Histoire, il est tout aussi important à présent de réinterroger les masculinités, qui ont trop longtemps été traitées comme allant de soi au sein de ce processus, et de problématiser les hiérarchies entre masculinités ${ }^{17}$. Les études critiques sur les masculinités s'emploient ainsi, dans le sillage des travaux de Raewyn Connell, à mettre en avant les tensions entre un modèle hégémonique de masculinité - historicisable - et les éventuels contremodèles ou anti-modèles ${ }^{18}$. Car à réduire la notion de « genre » à la seule catégorie des « femmes », on oublie le fait que toutes les masculinités ne jouissent pas de la même visibilité ni de la même reconnaissance sociale, politique et historique. Le genre, en tant que concept permettant d'analyser le social, fait advenir des régimes

Perspective ", in John PIER (dir.), The Dynamics of Narrative Form: Studies in Anglo-American Narratology, Berlin/ New York, De Gruyter (coll. « Narratologia »), 2004, p. 59-82; Ansgar NüNNING, «Unreliable, compared to what? Towards a Cognitive Theory of Unreliable Narration: Prolegomena and Hypotheses », in Walter GrüNzWEIG, Andreas Solbach (dir.), Grenzüberschreitungen: Narratologie im Kontext/ Transcending Boundaries: Narratology in Context, Tübingen, Narr, 1999, p. 53-73. Je remercie Anne Isabelle François pour ces références.

${ }^{16}$ Voir notamment Uta KLeIn, Militär und Geschlecht in Israel, Frankfurt a. M., Campus, 2001; Isabelle Lacoue-Labarthe, Femmes, féminisme, sionisme dans la communauté juive de Palestine avant 1948, Paris, L'Harmattan, 2012.

${ }^{17}$ Voir à ce sujet Anne-Marie SoHN (dir.), Une histoire sans les hommes est-elle possible?, Lyon, ENS Éditions, 2013.

18 Raewyn Connell, Masculinités. Enjeux sociaux de l'hégémonie [1995], trad. Maxime Cervulle, Marion Duval, Clémence Garrot, Claire Richard et Florian Voros, Paris, Éditions Amsterdam, 2014. 
de domination: c'est un instrument de pouvoir qui affecte tant les femmes que les hommes. La question n'est donc pas tant celle de la différence que celle du pouvoir.

\section{Le mensonge au cour de la construction du «nouvel homme juif »}

La question de la hiérarchie genrée des masculinités est, à mon sens, l'une des clefs de lecture pertinentes du roman 1948. Un premier niveau de lecture concerne les attributs du « nouveau juif», embrigadé en héros dans l'unité d'élite du Palmah ${ }^{19}$ pour défendre, les armes à la main, une terre et une nation juives en devenir. Ainsi les jeunes en Palestine mandataire sont-ils éduqués à une nouvelle virilité juive: « Ils [nos professeurs sionistes] voulaient que nous commencions à fabriquer une nouvelle mythologie juive, virile, qui serait nôtre, et que nous cessions de vivre aux crochets de l'Histoire d'autrui. » (34) Les figures pionnières du sionisme sont donc masculines ou, à tout le moins, masculinisées.

À première vue, le narrateur semble incarner tous les attributs de la masculinité de ce nouveau juif né en Eretz Israel. Il a eu une trajectoire archétypale : passé par des mouvements de jeunesse (14), il s'engage tout naturellement dans l'armée juive de défense en Palestine mandataire à l'âge de dix-sept ans et demi (15). Il se décrit luimême comme un enfant du pays, un Sabra, littéralement une " figue de barbarie ", soit un fruit du désert dur et piquant. Le texte distille à plusieurs endroits des indices de cet habitus et d'un répertoire corporel masculins spécifiques :

Car nous, nous étions nés dans ce pays. Dans les ronces. Avec les chacals. Avec [...] les figues de Barbarie, les grenadiers et les cyprès [...]. (17)

[C'était] la voix rocailleuse de l'Israélien de la génération de ceux qui croient que plus on crie, plus on a raison. (22)

[Nous étions de] charmants sabras hérissés de piquants, on nous avait créés ainsi afin d'être à l'opposé des Juifs de diaspora qu'on trouvait laids et niais. (47)

L'Israélien nouveau parlait hébreu et prônait le travail hébreu, nous voulions [...] féconder le désert, construire et se construire sur cette terre. (68)

S'opère donc une hiérarchie claire: la masculinité du «nouveau juif » s'oppose à la masculinité juive diasporique ${ }^{20}$; le « nouveau juif» est fort, beau, rusé (comme un « chacal ») et présente des rugosités.

Cette masculinité est celle qui doit mener à une conduite exemplaire et héroïque au service de la nation. Le narrateur remarque ainsi qu' «avec le conflit israélo-arabe ${ }^{21}$, le peuple juif a soudain eu besoin de héros. » (56) Allusion est faite

${ }^{19}$ Le Palmah (hébreu: פלמ"ח, acronyme de Plugot Mahatz, soit « unité de choc » ou «compagnie d'écrasement ») fut l'une des forces paramilitaires juives sionistes au sein de la Palestine sous mandat britannique. Il s'agissait de la formation d'élite de la Haganah et elle était constituée principalement de volontaires, notamment de kibboutzniks. Fondée en 1941, elle fut active pendant la Seconde Guerre mondiale et jusqu'en 1948, puis fut intégrée à l'armée israélienne.

${ }^{20}$ Au sujet de la soft masculinity du juif de la diaspora, voir Daniel BoYARIN, Unheroic Conduct: The Rise of Heterosexuality and the Invention of the Jewish Man, Berkeley, University of California Press, 1997.

${ }^{21}$ Des émeutes arabes de 1936 jusqu'à la première guerre israélo-arabe de 1948. 
ici, par exemple, à la redécouverte voire l'« invention » du mythe de Massada ${ }^{22}$, lieu de mémoire du combat acharné des juifs. Mais, évidemment, cet héroïsme viril ne tient pas à l'épreuve des faits. Ces derniers rendent l'héroïsme absurde et, du même coup, la virilité héroïque s'en trouve décrédibilisée: «Il faut être un parfait idiot - voire pire - pour marcher dans un champ de mines et croire qu'on est en train de sauver la Nation » (145), précise le narrateur. Et il insiste en évoquant la perte de semence virile et donc l'infertilité de ces actes qui se veulent masculins: "Nous sommes une goutte de semence perdue dans la grande vague qui a submergé les héros de cette armée, le Palmah » (246). Finalement, ces hommes ne partagent pas tant la semence noble dans un entre-soi de camaraderie virile qu'ils ne s'exercent à des concours de pets ou ne pissent ensemble sur des feux de camps pour occuper le temps: «Éteindre les bougies en pétant, j'en avais marre» (112), se lamente le narrateur en quête de sens. Et il ajoute un peu plus loin: « Je n'aimais pas m'exhiber en public comme tous les autres, qui pissaient en cercle pour éteindre les feux de camp. Moi, je restais toujours à l'écart, embarrassé » (114).

L'attitude du narrateur, qui se situe toujours un peu à l'écart des autres, sert en réalité de révélateur à une imposture : l'imposture de cette nouvelle masculinité juive. L'imposture est ici entendue comme une comédie sociale, comme la socialisation d'un mythe fantasmé ${ }^{23}$. Les souvenirs du narrateur viennent perturber la performance de genre célébrée dans la mémoire officielle. Le récit se révèle comme celui d'un antihéros détaché de la masculinité hégémonique. Il y a tout d'abord une conscience rétrospective aiguë du décalage formidable entre cette guerre et le destin des juifs en Europe quelques années auparavant. Cette comparaison émerge dans le récit au moment où le narrateur est confronté à l'arrivée des survivants de la Shoah en Israël après 1948, qui sont loin d'incarner le « nouveau juif » mais qui au contraire rappellent cruellement qu'il y a eu d'autres mondes juifs dans la diaspora:

Ces gens étaient sortis de l'enfer pour entrer dans l'Histoire. [...] À côté d'eux, nous faisions figure de plaisanteries ambulantes, de gogos imbus d'eux-mêmes qui avaient gagné une guerre de carton-pâte. Rien à voir avec ce qu'ils avaient connu, la Wehrmacht, les nazis, la Gestapo, les blindés, les wagons plombés, les blocs peints en gris et rejoindre Dieu par la cheminée des crématoires. (231-232)

Mais l'imposture ultime est aussi celle qui a rétrospectivement consisté, dans un entre-soi masculin ${ }^{24}$, à « laver » la guerre de 1948 de ses souillures. Le récit rappelle cruellement à quel point il ne s'agissait pas d'une guerre propre. Le point culminant de celui-ci est l'épisode où le narrateur, sous la pression du groupe masculin de camarades, tire sur un enfant arabe dans un village. L'enfant est tué. S'ensuit un récit qui imbrique deux voix qui débattent dans la tête du narrateur:

22 Voir à ce propos Mireille Hadas-Lebel, Massada, histoire et symbole, Paris, Albin Michel, 1995.

${ }^{23}$ Sur le concept d'imposture et ses liens avec la masculinité, voir Paul-Laurent Assoun, « L'imposture héroïque. L'art du semblant », Cliniques méditerranéennes, nº 81.1, 2010, p. 11-31.

${ }^{24}$ Bien que l'idéal sioniste puis la réalité militaire israélienne aient d'emblée accordé une place aux femmes, dans les faits, ces dernières ont été cantonnées à des postes « féminins » ainsi que l'ont montré les recherches en histoire des femmes. Voir Ilaria SimonetTI, « Le service militaire et la condition des femmes en Israël », Bulletin du Centre de recherche français à Jérusalem, n 17, 2006, p. 78-95. 


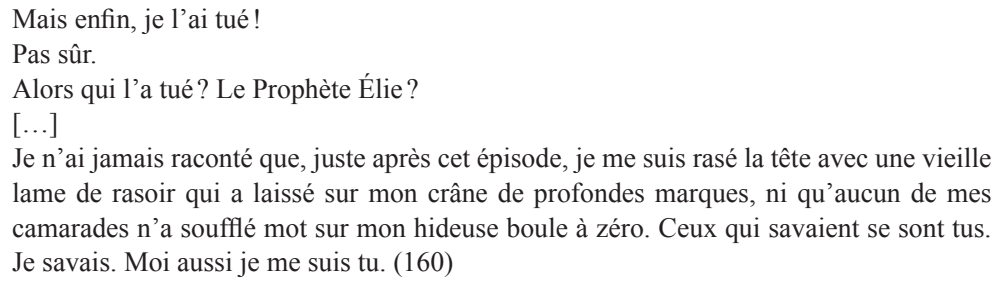

Le mensonge et l'imposture sont donc maintenus par le non-dit, dans l'entre-soi vétéran et masculin. Il s'agit là de cette "génération Palmah » sur laquelle s'est penchée l'historiographie critique israélienne ${ }^{25}$. Le narrateur de Yoram Kaniuk, lui-même membre - décalé - de la génération Palmah, brosse un portrait à contrecourant des hommes de sa génération, et constate l'engendrement de vétérans postmortem:

\begin{abstract}
Me souvenir - car sous peu il ne restera plus personne de ceux qui étaient avec moi... bien que, depuis un certain temps, je constate la prolifération des anciens combattants. Ceux d'alors se sont étrangement multipliés post-mortem. L'actuelle Maison du Palmah est beaucoup plus grande que ne l'a été le Palmah tout entier pendant sa période d'existence. Une « génération du Palmah » voit le jour, elle réalise des films sur le Palmah, organise des colloques sur le Palmah, nomme des comités de commémoration du Palmah. On attribue aussi des prix du Palmah et on réécrit l'histoire du Palmah - bravo, ils ont réussi à transformer le Palmah en entreprise florissante! (31-32)
\end{abstract}

Ce qui reste, c'est donc « un Palmah virtuel, une sorte de Club des Cinq pour adultes » où l'on célèbre une "fraternité » masculine chantée par le poète Haïm Gouri ${ }^{26}$ (245), et surtout, un business mémoriel. L'ouvrage de Kaniuk participe donc pleinement de la réflexion controversée autour des usages de la mémoire dans le contexte israélien ${ }^{27}$.

\title{
Le roman familial : identité juive-allemande et imposture paternelle
}

Le roman offre également un autre niveau de lecture se rapportant à la question de la masculinité : celui qui est centré sur une masculinité européenne-plus exactement juive-allemande et bourgeoise - incarnée par le père du narrateur. Cette masculinité «yekke »-du qualificatif servant à désigner les juifs allemands en Israël - apparaît

${ }^{25}$ Voir en particulier Anita SHAPIRA, Land and Power: The Zionist Resort to Force, 1881-1948, Oxford, Oxford University Press, 1992. Plus récemment, dans sa biographie du général et homme politique Yigal Allon, commandant du Palmah entre 1945 et 1948, Shapira dresse un portrait critique de l'ensemble de la « génération Palmah » (Yigal Allon, Native Son: A Biography, Philadelphie, University of Pennsylvania Press, 2008).

${ }^{26} \mathrm{Au}$ sujet de cette fraternité masculine des soldats (en hébreu «re'out»), voir Danny KaPLAN, The Men We Loved. Male Friendship and Nationalism in Israeli Culture, New York/ Londres, Berghahn, 2006, p. 20 sq.

${ }^{27}$ On peut faire le lien plus général avec le débat très controversé de l' « industrie de la mémoire » en Israël. Voir à ce sujet Idith Zertal, La Nation et la Mort. La Shoah dans le discours et la politique d'Israël, Paris, La Découverte, 2004. 
tout d'abord comme une alternative. L' « écart » permanent de «positionnalité » du narrateur par rapport à la masculinité hégémonique présentée ci-dessus lui vient peut-être - et c'est l'hypothèse que je formulerais ici - de cet héritage familial. Il est donc l'héritier d'une forme de masculinité bourgeoise de la "contention ${ }^{28}$ ", à l'opposé de l'idéal de masculinité soldatesque et militaire.

Arrivés en Palestine mandataire après 1933, les juifs allemands (Yekkes) ont eu à subir un stigmate important jusque dans les années 1960: celui de ne pas adhérer aux idéaux sionistes du «nouveau juif». «Kommen Sie aus Überzeugung oder kommen Sie aus Deutschland? », est l'un des nombreux calembours qui circulaient à leur sujet. Pour les hommes yekke, cela s'est parfois traduit par un stigmate de dévirilisation, lié au fait que parce qu'ils incarnaient un Bildungsbürgertum en exil, ils ne correspondaient pas aux attentes virilistes et pionnières du «nouvel Hébreu ». Pour le dire rapidement, la lecture de Schiller, Heine et Goethe n'était pas directement utile pour combattre les Arabes. Se réclamer d'une masculinité européenne, bourgeoise et cultivée, était donc une manière de se mettre à l'écart des injonctions genrées sionistes ${ }^{29}$. De même que le fils se met à l'écart des rites de camaraderie virile pendant la «Guerre d'indépendance », la performance de genre du père du narrateur est clairement identifiable comme étant celle d'un Yekke, qui met « une cravate même pour aller aux toilettes » ou qui nettoie « même les savons ${ }^{30}$ par souci de propreté » (41):

J'ai pensé à mon père qui pendant le Seconde Guerre mondiale, lorsque les Italiens bombardaient la ville, refusait de descendre à l'abri car, selon lui, d'après les statistiques, le risque d'être blessé à Tel-Aviv était d'un sur cinquante millions. Il restait donc assis dans sa chambre [...] à lire du Jean Paul ou du Heine. (27)

D'un autre côté, cette «Jeckischkeit » a aussi été un enjeu identitaire au sein de l'arène mémorielle israélienne. Le roman de Yoram Kaniuk révèle que l'identité yekke du père est en réalité un travestissement: c'est une dissimulation, un mensonge de plus afin d'apparaître comme ce que l'on n'est pas, notamment sur le plan ethno-social. Dans la constitution d'une mémoire yekke genrée en Israël, s'est en effet posée la question de la «vraie » Jeckischkeit - notamment par rapport à la Yiddishkayt ou à l'identité ostjüdisch. Dans le roman - qui reprend ici des éléments autobiographiques -, le narrateur met un point d'honneur à détruire la « couverture » que son père Moshe, premier conservateur du Musée de Tel Aviv (donc Yekke par la grâce de sa fonction, pour ainsi dire), s'est forgée tout au long de sa vie. Car ce père est en réalité un «Juif de Galicie qui se prenait pour un natif de Berlin et fredonnait parfois des prières hébraïques entre deux lieder de Schubert et de Brahms » (17).

${ }^{28}$ Sur l'apparition progressive d'un idéal de masculinité de la « contention » au cours du XIX ${ }^{\mathrm{e}}$ siècle, on se reportera à SoHn, «Sois un homme! ».

29 Concernant les injonctions à une masculinité hébraïque et pionnière dans le cinéma des années 1930 et 1940 en Palestine mandataire, voir Raz Yosef, Beyond Flesh. Queer Masculinities and Nationalism in Israeli Cinema, New Brunswick (NJ), Rutgers University Press, 2004, chap. 1.

${ }^{30}$ Un autre passage du roman rappelle par ailleurs cruellement que les juifs du yichouv (i. e. ceux qui constituaient la communauté juive de Palestine mandataire) surnommaient « savons » les survivants des camps d'extermination arrivés après 1945. « Nettoyer les savons » prend donc ici une connotation particulière. 
Une scène cruciale oppose ce faux Yekke à son cousin Ernst, Juif de Galicie survivant de la Shoah, qui est venu lui rendre une visite impromptue à Tel-Aviv. La question de la langue yiddish comme ancrage culturel y est centrale. Le cousin demande au jeune narrateur s'il parle cette langue. Bien évidemment, la réponse est négative, puisque le père Moshe, qui a emporté dans son exil ses disques de Beethoven en gage de sa Jeckischkeit, a tout fait pour mettre en avant le grand «passé allemand » de la famille. Il n'a transmis ni la langue ni la culture yiddish à son fils, qui répond: « Je lui dis alors que je regrettais de ne pas parler yiddish, il eut un sourire doucereux et répliqua, mais non et dis-moi, quand ti rêves, ti né rêves pas en yiddish? " (39) Le cousin Ostjude refuse de serrer la main au traître sabra: «D'autant qué jé réfise dé té serrer la main, espèce de bâtard sabra» (40). La rencontre s'envenime: "jé né palabré pas avec lé deutsche salaud dé ton père l'ordire » (40). Puis la scène tourne au cruel démasquage du père:

Et ton père, où il est né? À Berlin? Il est né à Ternopol, en Galicie, et s'il est autrichien, c'est iniquement parcé qué les salopards de François-Joseph sont arrivés jisque là, qu'il s'est batti pour eux, est dévéni officier et a vouli s'enrôler dans l'armée allemande. (44)

Les deux cousins en viennent aux mains, le masque yekke tombe et le narrateur entend son père parler yiddish pour la première fois :

Et soudain, ils s'empoignèrent et commencèrent à se battre [...] jusqu'au moment où le yiddish prit le dessus, et ce fut la première fois que j'entendis mon père parler cette langue, la première fois que je le vis frapper quelqu'un et la première fois que je le vis étreindre quelqu'un, car il ne serrait personne dans ses bras, ni sa femme, ni ma sœur, ni moi. (39-40)

La langue du passé ostjüdisch de la famille est donc toujours là. La langue du passé n'a été ni colonisée par l'allemand que le père a appris au cours de ses études à Berlin, ni balayée par l'imposition sioniste de l'hébreu moderne. Elle peut sans cesse resurgir dans l'inconscient, le rêve ou la perte de contrôle, et avec elle les enjeux européens de la mémoire juive de la galout ainsi que 1'hexis corporelle d'une autre masculinité, qui n'est ni la rigidité formelle et insensible yekke ni la virilité bruyante et rugueuse sabra. Ces deux dernières formes de masculinité sont alors renvoyées dos-à-dos, reflets toutes deux d'une société prônant des valeurs nationalistes agressives.

\section{Conclusion}

Le mensonge de genre qui se trouve au cœur du roman de Yoram Kaniuk, 1948, concerne la masculinité. Cette question a naturellement partie liée avec des enjeux historiques et mémoriels agitant la société israélienne. Le prisme du genre permet ici de traverser la question du sionisme, de la Shoah, de l'assimilation des juifs allemands ou encore de la morgue sociale de ces derniers vis-à-vis des Ostjuden. L'héroïsme affabulateur du Palmah, tout comme l'élitisme bourgeois juif allemand sont décrédibilisés et toute forme de tentative d'imposer une hégémonie du masculin paraît caduque. Ne reste, à l'issue du roman, que la trajectoire individuelle 
du narrateur, témoin aveugle et non-fiable de la période cruciale que furent les années 1945-1948:

Pendant toute la durée du combat, je n'ai pas réfléchi. Pas eu de projets. J'ai fait ce qu'on me disait de faire, je n'ai pris d'initiatives que lorsque je n'ai pas eu le choix et qu'il fallait improviser. On me disait de dormir, je dormais, on me disait de me lever, je me levais. On me distribuait de la nourriture, je mangeais. Si on ne me distribuait rien, je n'avais pas faim. Apparemment, on ajoutait du bromure dans notre ration d'eau, car je ne pensais pas aux filles alors qu'auparavant, leur féminité bourgeonnante me rendait fou. (13-14)

De ma peur, je suis sorti en héros qui a réussi à vaincre ses peurs. Avant, j'étais une boule d'angoisse. J'avais peur du noir. De la mort. Des gens. De la foule. (15) 